\title{
Integrated material process simulation for lightweight metal products
}

\author{
A. M. Horr \\ LKR Leichtmetallkompetenzzentrum Ranshofen GmbH, \\ Austrian Institute of Technology, Austria
}

\begin{abstract}
Over the past two decades, multi-physical material process simulation techniques have been developed and deployed using the powerful collection of experimental/numerical tools for the processing, characterization, and modeling of materials at different scales. These techniques, which require high-performance computers (parallel processing), can provide an unprecedented insight into processes of metal and alloys including multi-scaling phenomena (atomic-scale and molecular-scale structures). Using these tools, the synthesization, characterization, and numerical modelling of lightweight alloys and their composition can be performed (at the length/time scales). The casting and forming are popular production processes for light weight component with large industrial capacity, cost and energy savings. In numerical process simulations (i.e., casting, forming processes), the whole fluid (melt) domain along with thermal solidification, cooling system, microstructure and defect development have to be simulated. In the proposed integrated simulation technique, the heat transfer and solidification during casting process along large plastic deformation during forming has to be modelled accurately to predict the mechanical properties of the final product.
\end{abstract}

Keywords: material process simulation, integrated numerical methods.

\section{Introduction}

The production of high performance alloys is the main goal of material processes in lightweight metal industry underpinning the need for materials with better properties and lighter weights (lower inertia). The development of new lightweight alloys and their impact on low inertia mobility has been promoted in last couple 
of decades and special attention has been devoted to high performance lightweight alloys. In the research work herein, an attempt has been made to develop the required sophisticated tool for integrated simulation of material processes along with dynamic performance, corrosion analyses and micro-mechanical plasticity simulation. Some of the outcomes of the first stage of the research work have been presented in this paper, where the integration of Eulerian-based melt (thermalfluid) simulation has been carried out with a Lagrangian mechanical simulation (including defect modelling). One of the main contributions of this paper is to show the benefits of using integrated simulation techniques to model and optimize the material processes for lightweight alloys. A series of numerical/experimental programs has been setup to investigate these process and the results has been compared for verification study.

To perform a comprehensive process simulation of lightweight alloys for performance, due to its complicated multi-physical aspects, it is essential to use advanced and innovative numerical approaches. These methods should be capable of dealing with phase changes, thermal energy transfer, large deformation and also solid stress-strain state conditions. During the past two decades, numerical predictions of mechanical and damage and failure characteristics for lightweight components have been considered for the design of safer and lighter vehicles. Both continuous and discrete (discontinuous) numerical models can be used to simulate the chain of material processes where an efficient discretised numerical model can be developed. These two different approaches, namely; finite element/finite volume approach with large deformations and secondly, mesh-free/particle methods can both be used to simulate the fluid-thermal-mechanical material processes.

\section{Numerical techniques for material processes}

Many research works in the last couple of decades have attempted to use different mathematical and numerical methods to model the material processes as fluidthermal-mechanical processes (including large deformation process). The Eulerian, Lagrangian, Arbitrary Lagrangian $\backslash$ Eulerian, mixed Lagrangian $\backslash E u l e r i a n$, hybrid and mesh-less $\backslash$ particle techniques have been developed and used by different researchers to model the fluid-thermo-deformation process. In Lagrangian (updated Lagrangian with adaptive re-meshing) description the thermo-deformation process, the process of large deformation of a body is pictured as a material flow where each material particle (cell) carries its own properties such as density, etc. As the deformation front advances its properties may change in time. The procedure of describing the entire material flow by recording the detailed histories of each cell is the Lagrangian description. This means that in the Lagrangian algorithms, each individual node of the computational grid follows the associated material particle during the advance of extrusion front. Hence, The Lagrangian description allows an easy tracking of plastic deformation front (free surfaces) and interfaces between different parts of computational model. It also facilitates the treatment of materials with history-dependent constitutive relations, however, its main weakness is the inability of the method to 
follow large distortions of the computational domain without recourse to frequent re-meshing scheme (computationally expensive task).

While, in the Eulerian approach, rather than following each material cell (extrusion front), the evolution of the material flow properties at every point in space, as time varies, can be recorded. This means that the material flow properties at a specified location depend on its spatial location and on time. The computational mesh is fixed in this approach and the material moves with respect to the grid. In the Eulerian description, large distortions of the material motion can be handled with relative ease, but generally at the expense of precise interface definition and the resolution of material flow details. Since both of these methods have some shortcomings (updated Lagrangian and Eulerian methods), a hybrid technique has been developed that combines the best features of both the Lagrangian and the Eulerian approaches. In the arbitrary Lagrangian-Eulerian (ALE) description, the nodes of the computational mesh may be moved with the material front in normal Lagrangian fashion, or fixed in Eulerian manner or be moved in some arbitrarily specified way to give a continuous rezoning capability. More comprehensive discussion about the Lagrangian, Eulerian and ALE approaches can be found in engineering handbooks [1].

\subsection{Casting process simulation}

To achieve a required product quality during early processes in the chain of production lines, it is necessary to identify and also control the main input parameters affecting the casting defects to arrive at the desired output quality. In industrial scale casting, where the issues of part quality, cost saving and cast speed are the main driving forces for the industries, optimization of the cast process to have minimum defects is quit essential. The popular casting processes of light weight metals are defined as a process where the die is filled by relatively low pressure (LPDC), high pressure (HPDC), squeezed, continuous and semicontinuous (see fig. 1) melt casting. In recent years, in order to limit the product defects, novel through process simulation methods have been employed to investigate casting parameters including basic alloy components, melt flow, casting thermal conditions and cooling rates.
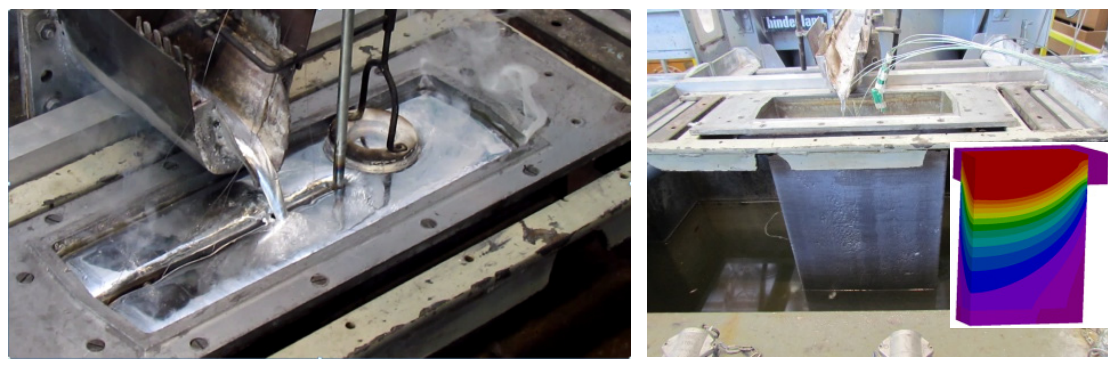

Figure 1: Semi-continuous casting of alloy and its simulation. 
Recent advances in numerical techniques have encouraged full multi-physical (thermal-fluid-solid) simulations of casting process, for industrial and also laboratory scale productions, as one of the important parts in controlling the material quality, design and fabrication. The complex aspects of casting process including its multi-physical dimension (fluid flow, cooling rates, air gaps and mechanical stress) as well as material phase changes, makes the process simulation awkward. The casting material characteristics (i.e., workability, strength, durability, fatigue) would depend on the heat and mass transfer during casting process and the possible further heat treatment process. The solidification process and its complex phase changing nature demand an equally sophisticated numerical methodology for the cast process simulation. It involves liquid-mushy-solid multi-phase changes where the thermal conduction and convection plays an important role. The numerical approach towards the solidification modelling can simply start with considering the conduction-based energy transfer mechanism. However, the effect of mass transfer and convection cannot be ignored for many cast process simulations. The heat energy transfer during solidification is calculated based on conduction-convection-radiation heat transfer. To account for the convection heat transfer effects, a simple artificial high fluid conductivity can be defined for initial estimation.

The more precise approach is to account for convection explicitly in fluid equations. The mass transfer and thermal convections are taken into account using governing differential equations for the melt flow. The enthalpy of a system can be written [2] as $H=h+\Delta H$, where $h$ is called sensible enthalpy $\left(h=h_{\text {ref }}+\right.$ $\left.\int_{T_{\text {ref }}}^{T} c_{p} d T\right)$ and $h_{\text {ref }}, T_{\text {ref }}, \Delta H$ and $c_{p}$ are reference enthalpy, reference temperature, latent heat and specific heat. The latent heat for each element in the mesh is assigned, in this method, and the corresponding energy equation for the element is adjusted for the nodal heat values. The momentum equation should also be damped at solidifying front where the velocity field (for melt) needs to be reduced to zero for the material in solid state. Different methods have been proposed in the literature to damp the momentum equation at solidifying front. A practical method of damping the momentum equation is proposed by Voller et al. [2] in which the solidifying front is modelled as a pseudo porous media. This enthalpy-porosity approach is a reliable way to numerically simulate the solidification process through the definition of liquid fraction $\beta$ in the mushy as: $\beta=0$ if $\mathrm{T}<\mathrm{T}_{\text {solidus, }} \quad \beta=1$ if $\mathrm{T}>\mathrm{T}_{\text {liquidus }}$ and

$$
\beta=\frac{T-T_{\text {solidus }}}{T_{\text {liquidus }}-T_{\text {solidus }}} \quad \text { if } \mathrm{T}<\mathrm{T}_{\text {solidus }}
$$

After defining the liquid fraction at the solidifying front, the energy equation can be written as:

$$
\frac{\partial \rho H}{\partial t}+\operatorname{div}\left(\rho u_{v} H\right)=\operatorname{div}[\alpha \cdot \operatorname{grad}(T)]+S_{s}
$$

where $\alpha, u_{v}, \rho$ and $S_{s}$ are thermal diffusivity, velocity vector, density and energy source/sink and the mathematical operators div and grad are diversion and gradient operators. There are different numerical strategies for the implementation of phase changes in fluid-thermal, fluid-thermal-mechanical or even thermalmechanical process simulations. For the transient thermal-mechanical behavior of 
casting process, the simulated can be carried out using Lagrangian finite element analyses with element-based coupled formulation. The objective of this type of simulation is to predict the thermal gradient, spatial temperature mapping and stress-strain response of the cast alloys during the continuous casting process. Transient non-linear thermal-mechanical finite element analyses are employed in this simulation approach using element based coupled solver and moving mesh technology.

The pure Eulerian, Arbitrary Lagrangian-Eulerian (ALE) and Mixed Lagrangian-Eulerian (MiLE) formulations have also been used in recent decades to simulate the casting process as an alternative to the Lagrangian-based simulation methods. Many of the current commercial cast process simulation tools are based on either Eulerian, MiLE or ALE computational techniques. In MiLE cast process approach, the analysis domain can be split into three mesh zones; Eulerian mesh zone where the melt flow would be simulated, Lagrangian mesh zone for solidified alloy, and finally, unfolding mesh zone to simulate the new layers (see fig. 2) of mesh for dynamic part of the casting (continuous casting). The method has produced reasonable results, although the issues of varying Heat transfer coefficient (HTC) at boundaries and also thermal heat loss variation (e.g., with an air gap) are still needed to be resolved.

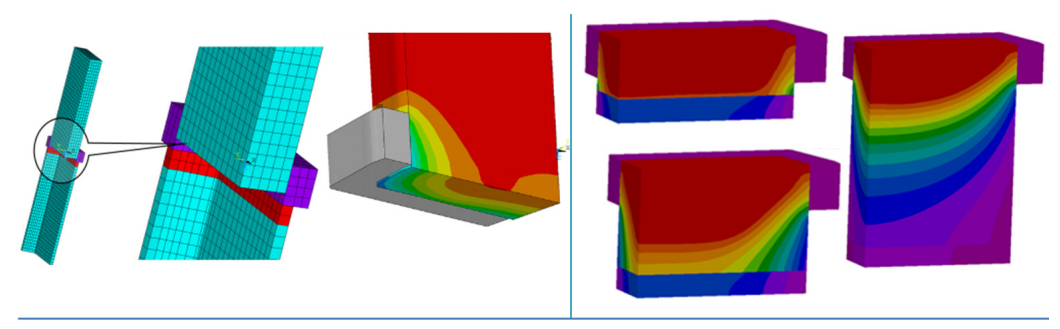

(a)

Figure 2: (a) Moving-mesh Lagrangian and, (b) Mixed Lagrangian-Eulerian simulations of semi-continuous casting for lightweight alloy.

In the study herein, a novel coupled (fluid-thermal-mechanical) dynamic mesh technique has been developed to improve the simulation technique for continuous casting process. Consider the governing conservation equations for prediction of compressible Navier-Stokes equations as:

$$
\frac{d}{d t} \int_{V} \rho \varphi d V+\int_{\partial V} \rho \varphi\left(u-u_{g}\right) \cdot d A=\int_{\partial V} \Gamma \nabla \varphi \cdot d A+\int_{V} S_{\varphi} d V
$$

where $\rho$ and $u$ are the fluid density and velocity respectively, $u_{g}, S_{\varphi}, \varphi$ and $\mathrm{V}$ are the velocity of moving mesh, the source term, conservation variable and a control volume with moving boundaries respectively. The time varying conservation term in eqn (3) can be numerically calculated using backward difference method,

$$
\frac{d}{d t} \int_{V} \rho \varphi d V=\frac{(\rho \varphi V)^{n+1}-(\rho \varphi V)^{n}}{\Delta t}
$$


The change in the control volume during the simulation can be written as,

$$
V^{n+1}=V^{n}+\frac{d V}{d t} \Delta t=V^{n}+\left(\int_{\partial V} u_{g} \cdot d A\right) \Delta t=V^{n}+\left(\sum_{j}^{f} u_{g, j} A_{j}\right) \Delta t
$$

where $A_{j}$ is the face area vector and $f$ is the number of faces on the control volume. For this study, a finite volume dynamic mesh method has been employed as [3],

$$
\frac{d}{d t} \int_{V} \varphi d V=\int_{V} \frac{\partial \varphi}{\partial t} d V+\int_{V} \varphi V_{j} d n_{j}
$$

where $V_{j}$ is the velocity of boundary (vehicle) and $d n_{j}$ are the differential Cartesian components of the outward normal surface vector. To assess the efficiency and accuracy of the method, an axisymmetric model of a semi-continuous casting process has been developed for a round straight billet. The two dimensional geometry has been meshed with structured axisymmetric elements for transient simulation. A series of novel user define routines have been written in $\mathrm{C}++$ language to generate and unfold new element layers at the bottom of the mold (inference of mold and billet domains). These $\mathrm{C}++$ routines have been complied and linked to FLUENT [4] software through Dynamic Link Library (DLL). They can be called upon in the Fluent user interface page, where they would be assigned as boundary conditions for domain interfaces. These user routines can unfold new layers of structured mesh at the interface, simulating continuous movement of the billet during casting. The sizes and frequency of the unfolded mesh layers need to be specified to start adding them into the domain.

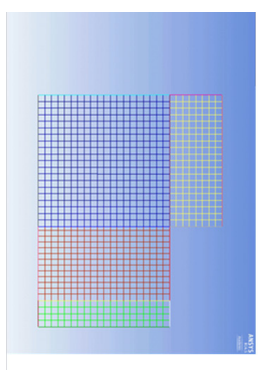

(a)
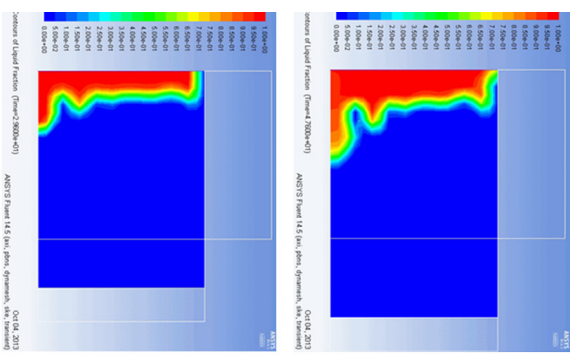

(b)

Figure 3: Coupled (fluid-thermal-mechanical) dynamic mesh technique, (a) dynamic unfolding mesh and, (b) melt volume fraction contour.

\subsection{Forming process simulation}

The Eulerian, Lagrangian, ALE and mesh-less techniques have been developed and used by different researchers to model the thermo-large-deformation processes. However, the Lagrangian (updated Lagrangian with adaptive remeshing) is suffering from the inability to follow large distortions of the computational domain without recourse to frequent re-meshing scheme (computationally expensive task). In the Eulerian approach rather than following each material cell, the evolution of the material flow properties at every point in 
space, as time varies, can be followed. Although, the large distortions of the material motion can be handled with relative ease in this technique, it is generally carried out at the expense of precise interface definition and the resolution of material flow details. The hybrid ALE and MiLE approaches treats the large deformation by separating grids or moving grid (in some arbitrarily specified way), but handling strong discontinuities is still a challenge.

The strong discontinuities which are natural consequence of the some forming processes encourage the use of numerical grid where large distortion can be handled easily. The most practical strategy for dealing with these moving discontinuities is to carry out adaptive re-meshing of grid nodes in each computational step (i.e., meshless). The evolution of new computational grid is carried out so that mesh lines remain coincident with the discontinuities throughout the evolution of transient extrusion front. The goal of meshless technique is to remove the numerical constraints of computational grid by constructing an approximation kernel based on entire points (nodes). Although in many meshless methods recourse is required to be taken to some type of computational grid in the course of transient simulation, the moving discontinuities can usually be modelled without re-meshing (which is computational expensive). This would open the way for the efficient solution of large classes of process problems which are very naturally awkward with gridbased methods.

The meshless Element-Free Galerkin (EFG) technique, which is capable of handling process simulation with arbitrary and complex geometries using only nodal data, has gain popularity in recent years $[5,6]$. The method can be applied to elasticity, heat conduction, large deformation and fracture mechanical problems with much less computational efforts. The EFG technique is developed using the principal of diffuse element technique [5] which has been introduced earlier for generalising the finite element method. For EFG technique, Moving Least-Squares (MLS) interpolants are used to construct a function for the variational principle (weak form) where dependent variable and its gradient are continuous in the entire domain [5]. The MLS interpolant of displacement function $u^{h}(x)$ can be written as [5],

$$
u^{h}(x)=\sum_{j}^{m} p_{j}(x) a_{j}(x)=\boldsymbol{P}^{T}(\boldsymbol{x}) \boldsymbol{a}(\boldsymbol{x})
$$

where $p_{1}(x)=1$ and $p_{j}(x)$ are polynomial functions (with single term) in the coordinate system. For the two dimensional quadratic basis function $\boldsymbol{P}^{T}(\boldsymbol{x})=$ $\left[1, x, y, x^{2}, x y, y^{3}\right], m=6$ and the coefficients $a_{j}(x)$ are fuctions of $x$. The $\boldsymbol{a}(\boldsymbol{x})$ can be obtained at any coordinate point $x$ by minimizing a weighted discrete norm,

$$
J=\sum_{i}^{n} w_{a}\left(\boldsymbol{x}-\boldsymbol{x}_{\boldsymbol{i}}\right)\left[\boldsymbol{P}^{\boldsymbol{T}}\left(\boldsymbol{x}_{\boldsymbol{i}}\right) \boldsymbol{a}(\boldsymbol{x})-u_{i}\right]^{2}
$$

where $n$ is the number of proximity points which the weighted function $w_{a}(\boldsymbol{x}-$ $\left.\boldsymbol{x}_{\boldsymbol{i}}\right) \neq 0$ and $u_{i}$ is the nodal value of $u$ at $\boldsymbol{x}=\boldsymbol{x}_{\boldsymbol{i}}$. For each of the nodes in the computational domain, the domain of influence (kernel influence) is defined by a circle (in two dimensional) of neighbourhood nodes. The numerical implementation of the meshless EFG technique for extrusion application can be started by defining influence sub-domains for each node as circle (two dimensional) and sphere (three dimensional) in the active coordinate system as shown in Table 1. 
Table 1: Definition of weighted function.

$w_{a}\left(\boldsymbol{x}-\boldsymbol{x}_{I}\right)=\left\{\begin{aligned} \text { for } 0 \leq \frac{\left\|\boldsymbol{x}-\boldsymbol{x}_{I}\right\|}{a} \leq \frac{1}{2} & \Rightarrow \frac{2}{3}-4\left(\frac{\left\|\boldsymbol{x}-\boldsymbol{x}_{I}\right\|}{a}\right)^{2}+4\left(\frac{\left\|\boldsymbol{x}-\boldsymbol{x}_{I}\right\|}{a}\right)^{3} \\ \text { for } \frac{1}{2}<\frac{\left\|\boldsymbol{x}-\boldsymbol{x}_{I}\right\|}{a} \leq 1 & \Rightarrow \frac{4}{3}-4\left(\frac{\left\|\boldsymbol{x}-\boldsymbol{x}_{I}\right\|}{a}\right)+4\left(\frac{\left\|\boldsymbol{x}-\boldsymbol{x}_{I}\right\|}{a}\right)^{2}-\frac{4}{3}\left(\frac{\left\|\boldsymbol{x}-\boldsymbol{x}_{I}\right\|}{a}\right)^{2} \\ \text { otherwise } & \Rightarrow 0\end{aligned}\right.$

The above mentioned functions are called the B-spline kernel function which defines the weighting function for each point in the computational domain. The graphical representation [5] of the technique is shown in fig. 4.
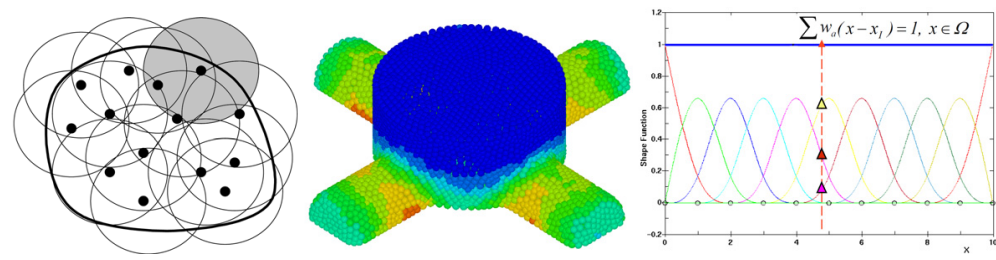

Figure 4: Sub-domains definition, nodes 3D representation and Spline kernel functions for EFG.

More comprehensive discussion about the EFG technique can be found in engineering literature $[5,6]$. In the research work herein, a comprehensive experimental/numerical study has been carried out to employ an efficient numerical meshless technique for extrusion processes and compare the results with carefully-setup experimental trials. A mechanical macro model of damage and failure prediction has also been developed to enable a performance simulation run for final lightweight components.

\section{Numerical damage modelling - macro model}

The final part of the material process chain simulation is the macro-mechanical numerical model, where a performance simulation is carried out for the final product. Based on the nonlinear transient dynamic theory and theory of damage accumulation and failure, as the loading condition is changing for the material, a plastic deformation may take place which would progressively increase the damage in the component. The accumulated damage would ultimately result in the failure of the cross-section. There are different numerical models to calculate the damage evolution, fracture initiation and also its propagation using continuum and/or discrete damage techniques [7, 8].

The nonlinear dynamic analysis and vibration effects along with energy absorption of crash-capable lightweight components (through stable folding), has attracted many researchers attention in recent years. The explicit finite element 
technique has been extensively used in nonlinear transient dynamic simulations for crash analysis. The size of time steps for explicit integration is limited by the numerical stability of the analysis. Hence, an explicit dynamic simulation using conventional finite element method usually employs many small steps. For the explicit dynamic analysis the weak form of the momentum equation can be written as,

$$
\int_{\Omega} \rho \ddot{u}_{i} v_{i} d \Omega+\int_{\Omega} \sigma_{i j} \frac{d v_{i}}{d x} d \Omega=\int_{\Omega} f_{i} v_{i} d \Omega+\int_{\Gamma_{S}} g_{i} v_{i} d \Omega
$$

where $u_{i}$ and $v_{i}$ are the displacement and variation components, $\sigma_{i j}$ are the stress components, $\rho$ is the mass density, $f_{i}$ are the components of body forces per unit volume, $g_{i}$ are the components of surface tractions specified on part of the boundary $\Gamma_{s}$ for the domain $\Omega$. More comprehensive discussion about the nonlinear explicit dynamic simulation technique can be found in engineering handbooks [1].

The development of proper material, damage and failure modelling is one of the first requirements for successful macro-mechanical simulation. One of the first pioneers in continuum damage mechanics were Rice and Tracy [9]. Their damage models were based on mathematical formulation for creep and spherical voids in continuum materials. Gurson [10] has extended the theory of damage evolution, using spherical voids in a continuum, by investigating the evolution of ductile damage due to growth and nucleation of voids in an elasto-plastic material. The more recent damage model is proposed by Lemaitre [11] in which a micromechanical model is developed to simulate the void growth, nucleation and coalescence in continuum. A "Generalized Incremental Stress State dependent damage Model" (GISSMO) has also been proposed by Neukamm et al. [12] recently. It is based on combination of incremental material instability, failure description and localization.

There are different ways of modelling reversible and irreversible deformations in the numerical simulation using mathematical theories. The yield criterion is one of the most popular methods of determining the state of stress and strain in the metals. For the study herein, a yield criteria based on the recent work by MATFEM [13] has been used as,

$$
\begin{gathered}
\sigma_{e q}=k_{1} \cdot\left(\left|X_{1}-X_{2}\right|^{m_{1}}+\left|X_{2}-X_{3}\right|^{m_{1}}+\left|X_{3}-X_{1}\right|^{m_{1}}\right)^{\frac{1}{m_{1}}}+k_{2} \cdot\left(\left|Y_{1}\right|^{m_{2}}+\right. \\
\left.\left|Y_{2}\right|^{m_{2}}+\left|Y_{3}\right|^{m_{2}}\right)^{\frac{1}{m_{2}}}
\end{gathered}
$$

where $X_{1}, X_{2}$ and $X_{3}$ are the principal components of the stress vector $\mathrm{X}$ which can be calculated from the stress tensor $\sigma$ and coefficient matrix $\mathrm{C}$ as described in MATFEM manual [13]. The formulation requires the definition of fifteen independent parameters $\left(m_{1}, m_{2} \ldots\right)$ for the yield locus in the general stress state. For the plane stress state (constant or no stress in third direction), the number of independent parameters are reduced to eleven. To account for tensioncompression asymmetry condition, the yield locus would be adjusted depend on the stress state (tensile or compressive stress) as;

$$
\sigma_{e q}=\sigma_{e q}(\sigma, k) \quad k=k\left(\sigma, k_{T}, k_{G}\right)
$$

where the set of coefficient $k_{T}$, will be used for tension stress state $\left(\sigma_{3} \geq 0\right)$ and the set of coefficient $k_{C}$, would be used for compression case. For other cases 
(second and forth quadrants) the coefficients would be interpolated to reach at a set of tension-compression coefficients.

The hardening curve can be applied to the material model using true stress and strain $\left(\varepsilon_{t}=\ln \frac{L_{1}}{L_{0}}\right.$ and $\sigma_{t}=\sigma_{e n g} e^{\varepsilon_{t}}$ where $\sigma_{e n g}$ is the engineering stress). It is well known that the hardening properties of materials can be isotropic, anisotropic or kinematic depend on the intrinsic plastic (slip) material behaviour. Different analytical strain hardening rules can be used to describe the evolution of yield locus and flow potential in plastic zones. The material model used herein is capable of taking into account the isotropic, kinematic and anisotropic as well as combination of isotropic-kinematic hardening.

\section{Integrated process simulation}

Recently, the use of lightweight alloys (aluminium and/or hybrid aluminiummagnesium alloys) for the mobility applications (i.e., design of electric and hybrid vehicles) have gained popularity, and special attention has been devoted to full through material process simulations for these products. To assess the overall performance of lightweight formed components including strength, dynamical, fatigue and damage, a full through process simulation is required to model the material properties from start of alloy process (i.e., casting) to the final forming and assembly. In the study herein, a framework has been setup to carry out an integrated through process simulations for lightweight mobility part. A casting simulation using fluid-thermal-mechanical approach followed by an extrusion simulation using EFG technique and finally a transient dynamic simulation have been performed. The casting simulation is carried out using novel dynamic mesh technique where new layers of elements have been generated during casting process and new source of energy is introduced in each step. For the extrusion simulation (see fig. 5), both ALE and EFG techniques have been employed and results are compared.

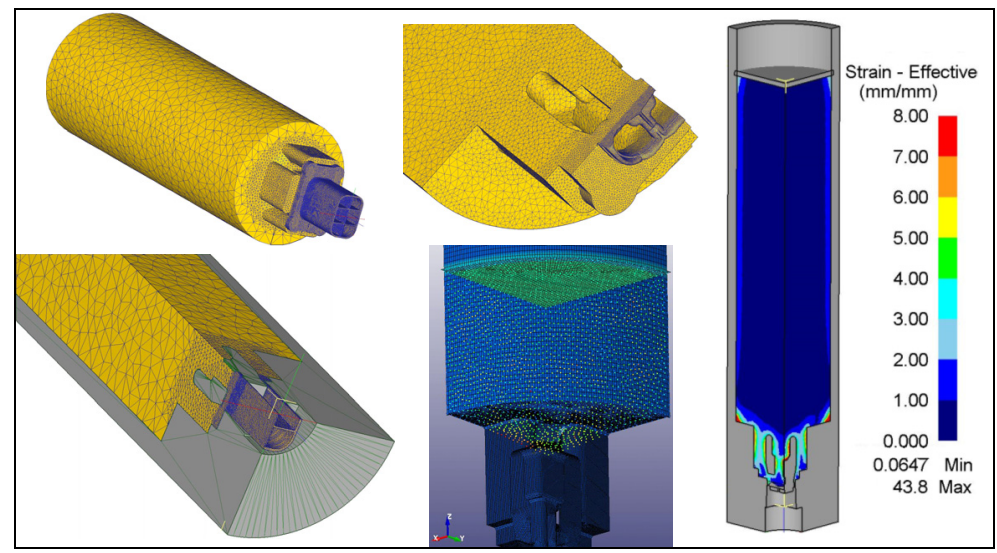

Figure 5: Mesh, node could (EFG) and effective strain results for extrusion process simulation. 
In the final part of the simulation chain, a dynamic simulation and virtual crash performance of vehicle components (crash-capable and energy absorbers) have been carried out for the design of lightweight vehicles components (see fig. 6).
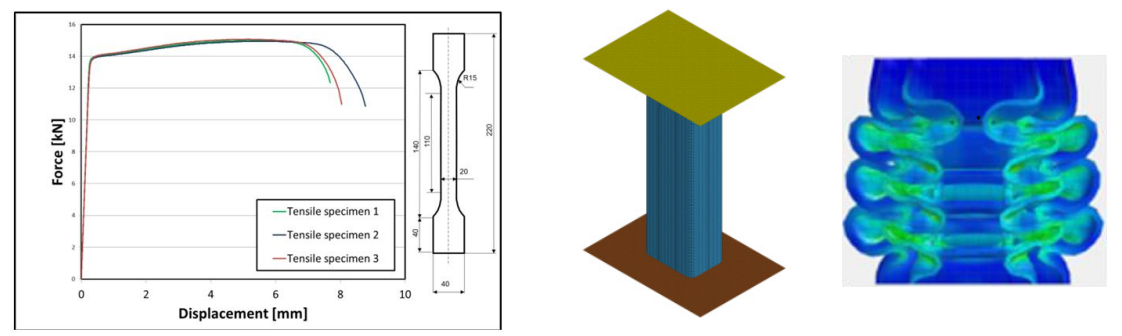

Figure 6: Tension and crash experimental tests.

\section{Concluding remarks}

An integrated numerical approach for the material through process simulation of light weight components has been presented herein. The combination of casting, forminglextrusion and mechanical process simulations and their implementations have been study for the performance-based design of new components. A series of experimental $\backslash$ simulation tests have also been carried out for selected extruded profiles to verify the simulation technique. The comparative analyses between the experimental and simulation results show that this upgraded numerical technique, have produced reasonable predictions for the dynamic, damage and failures in light weight extruded profiles.

\section{Acknowledgements}

The authors would like to thank the Austrian Federal Ministry for Transport, Innovation and Technology (bmvit) and also the Austrian Institute of Technology (AIT) for their financial support. The author would also like to thank, DI Matthias Hartmann, DI Johannes Kronsteiner, DI Florian Grabner and DI Andreas Kraly of Leichtmetallkompetenzzentrum Ranshofen GmbH, AIT, for their contributions.

\section{References}

[1] Bathe, K. J., Finite Element Procedure, Prentice Hall Inc, Upper Saddle, New Jersey, USA, 1996.

[2] Voller, V. R., Markatos, N. C., and Cross, M., Solidification in Convection and Diffusion, Numerical Simulation of Fluid Flow and Heat/Mass Transfer Processes, Springer-Verlag, Berlin, 1986.

[3] M. Griebel, T. Dornseifer and T. Neunhoeffer, Numerical Simulation in Fluid Dynamics, a Practical Introduction. SIAM, Philadelphia, 1998.

[4] FLUENT 14, Ansys Inc., 10 Cavendish Court, Lebanon, NH, 2012. 
[5] Belytschko, T., Krongauz,Y., Organ, D., Fleming, M. and Krysl, P., Meshless methods: an overview and recent developments, Computational Methods in Applied Mechanics Engineering, Vol. 139, pp. 3-47, 1996.

[6] Chen, J.S., C. Pan, Wu, C.T. and Liu, W.K., Reproducing Kernel Particle Methods for Large Deformation Analysis of Nonlinear Structures, Comput. Methods Appl. Mech. Engrg. 139, pp. 195-227, 1996.

[7] Horr,A. M., Damage and Failure Modelling of Aluminium Alloys, LKR internal progress report, pp. 4-15, 2013.

[8] Angermeier, C., Horr, A. M., Kretz, R., An innovative use of CT method in light metals development, ICT2014 - 5th Conference on Industrial Computed Tomography, pp. 149-157, 2014.

[9] Rice, J.R. and Tracy, D.M., On ductile enlargement of voids in triaxial stress fields, J. of Mechanics and Physics of Solids, 17, pp. 210-217, 1969.

[10] Gurson, A. L., Continuum theory of ductile rupture by void nucleation and growth, Part I: Yield criteria and flow rule for porous ductile media. J. Eng. Material Technology, Vol. 99, pp. 1-15, 1977.

[11] Lemaitre, J., A continuous damage mechanics model for ductile fracture, J. of Engineering Materials and Technology, 107, pp. 83-89, 1985.

[12] Neukamm, F., Feucht, M. and Haufe, A., Considering damage history in crashworthiness simulation, Ls-Dyna Anwenderforum, 2009.

[13] MATFEM User Manual, MATFEM Partnerschaft, München, Germany, 2008. 